PROCEEDINGS OF THE

AMERICAN MATHEMATICAL SOCIETY

Volume 126, Number 1, January 1998, Pages 75-84

S 0002-9939(98)03506-0

\title{
SUBLATTICES OF THE BANACH ENVELOPE OF WEAK $L^{1}$
}

\author{
HEINRICH P. LOTZ AND N. T. PECK
}

(Communicated by Dale E. Alspach)

\begin{abstract}
We prove that every separable Banach lattice is lattice isometric to a closed sublattice of the Banach envelope of Weak $L^{1}$.
\end{abstract}

For a finite nonatomic separable measure space $(X, \Sigma, \mu)$ we study lattice isometric and order isometric embeddings into the Banach envelope $\mathbb{W}$ of the Lorentz space $L(1, \infty)$. It was shown in [4] that $L^{1}$ and $\ell^{\infty}$ are lattice isometric to closed sublattices of $\mathbb{W}$, and it was shown in [9] that a separable Banach lattice with order continuous norm is lattice isometric to a closed sublattice of $\mathbb{W}$. Using a different approach than in [4] and [9], we show in this paper that every separable Banach lattice is lattice isometric to a closed sublattice of $\mathbb{W}$ and that $L^{\infty}$ as well as Weak $L^{p}, 1<p<\infty$, of a separable measure space is lattice isometric to a closed sublattice of $\mathbb{W}$. See also [7], in which the first author has given a characterization of the dual space of $L(1, \infty)$.

Let $(X, \Sigma, \mu)$ be a measure space. The Lorentz space $L(1, \infty)$ consists of all (equivalence classes of) $\mu$-measurable functions $f$ on $X$ for which

$$
\||f|\|=\sup _{s>0} s \mu\{|f|>s\}<\infty .
$$

This space with the topology defined by the quasinorm $\mid\|\cdot\|$ is called Weak $L^{1}$. Let $U$ be the convex hull of $V=\{f:\||f|\| \leq 1\}$ and let $\|\cdot\|$ be the Minkowski functional of $U$. Since $V$ is solid, i.e., $f \in V,|g| \leq|f|$ implies $g \in V, U$ is solid. Hence $\|\cdot\|$ is a lattice seminorm on $L(1, \infty)$ and $\|\cdot\| \leq\|\cdot\|$. Let $I$ be the ideal $\{f:\|f\|=0\}$. Then $L(1, \infty) / I$ with the quotient order and the norm $\|f+I\|=\|f\|$ is a normed vector lattice. Its completion $\mathbb{W}$ is a Banach lattice, which is called the Banach envelope of Weak $L^{1}$. By identifying $f$ with $f+I$, we can identify Weak $L^{1}$ with a dense sublattice of $\mathbb{W}$.

It has been shown in [1] and [2] that in the nonatomic case

$$
\|f\|=\limsup _{q / p \rightarrow \infty} \int_{p<|f| \leq q}|f| d \mu / \ln \frac{q}{p} .
$$

We will only consider Weak $L^{1}$ and its Banach envelope in the case where $(X, \Sigma, \mu)$ is a finite nonatomic separable measure space. Thus, we can restrict ourselves to the case $X=(0,1]$ and $\mu=\lambda$, where $\lambda$ denotes Lebesgue measure on

Received by the editors March 13, 1995.

1991 Mathematics Subject Classification. Primary 46B30, 46 E30.

Key words and phrases. Banach lattice, Banach envelope, Lorentz space, Weak $L^{1}$, Weak $L^{p}$, lattice isometry, order isometry. 
$(0,1]$. Throughout this paper, $\lambda$ denotes Lebesgue measure on $(0,1]$ and $L^{1}$ denotes the space $L^{1}((0,1], \lambda)$.

Let $E$ and $F$ be Banach lattices. A linear operator $T$ from $E$ into $F$ is called a lattice homomorphism if $|T x|=|x|$ for every $x \in E$. If, in addition, $\|T x\|=\|x\|$ for all $x \in E$, then $T$ is called a lattice isometry.

1

We start with embeddings of $L^{1}$ and $L^{\infty}(0, \infty)$ into Weak $L^{1}$ and its envelope $\mathbb{W}$. We use the unit square $X=(0,1] \times(0,1]$ with Lebesgue measure $\mu$ rather than the unit interval (cf. g) of the proof of Theorem 1 below).

Proposition 1. For $f \in L^{1}$ define $T f$ by

$$
(T f)(s, t)=f(t) / s, \quad(s, t) \in X .
$$

Then $T$ is a lattice isometry from $L^{1}$ into the Banach envelope of Weak $L^{1}$ on $X$ and a lattice "isometry" into the quasinormed space Weak $L^{1}$.

Proof. An easy calculation shows that

$$
\|f\|=\sup _{p>0} p \int_{0}^{1} \int_{0}^{\frac{\mid f(t \mid)}{p} \wedge 1} d s d t=\|f\|
$$

and that

$$
\|T f\|=\limsup _{\substack{q / p \rightarrow \infty \\ 0<p<q}} \int_{0}^{1} \int_{\frac{|f(t)|}{q} \wedge 1}^{\frac{|f(t)|}{p} \wedge 1} \frac{|f(t)|}{s} d s d t / \ln \frac{q}{p}=\|f\| .
$$

Proposition 2. There exists a lattice homomorphism $T$ from the Banach lattice $L^{\infty}(0, \infty)$ into Weak $L^{1}$ with

$$
\|T f\|=\|T f\|=\|f\|
$$

for all $f \in L^{\infty}$.

Proof. Let $\left\{\left(u_{n}, v_{n}\right]: u_{n}<v_{n}\right\}$ be an enumeration of the subintervals of $(0, \infty)$ with rational endpoints. Define $\psi_{n}:(0,1] \rightarrow\left(u_{n}, v_{n}\right]$ by $\psi_{n}(t)=u_{n}+\left(v_{n}-u_{n}\right) t$. For $f \in L^{\infty}$ let $f_{n}=f \circ \psi_{n}$. Then

$$
\|f\|=\lim \sup \int\left|f \circ \psi_{n}\right| d \lambda .
$$

Choose $0<a_{n}<b_{n} \leq 1$ with $b_{n+1}<a_{n}$ and $\lim \frac{b_{n}}{a_{n}}=\infty$. For $f \in L^{\infty}$ and $0<s, t \leq 1$ define

$$
(T f)(s, t)= \begin{cases}\frac{f_{n}(t)}{s} & \text { for } a_{n} \leq s \leq b_{n}, \\ 0 & \text { for } s \notin \cup\left[a_{n}, b_{n}\right] .\end{cases}
$$

Suppose that $f \in L^{\infty}$ and that $\|f\|=1$. Then $|(T f)(s, t)| \leq 1 / s$ and hence $\|T f\| \leq 1$. Fix $0<\delta<1$ and let $p_{n}=\delta / b_{n}$ and $q_{n}=1 / a_{n}$. If $a_{n} \leq s \leq b_{n}$ and 
$\delta<|f(t)|$, then $p_{n}<|(T f)(s, t)| \leq q_{n}$. Hence

$$
\begin{aligned}
\int_{p_{n}<|T f| \leq q_{n}}|T f| d \mu & \geq \int_{\left|f_{n}\right|>\delta} \int_{a_{n}}^{b_{n}} \frac{\left|f_{n}(t)\right|}{s} d s d \lambda(t) \\
& =\int_{\left|f_{n}\right|>\delta}\left|f_{n}\right| d \lambda\left(\ln \frac{q_{n}}{p_{n}}+\ln \delta\right) .
\end{aligned}
$$

Therefore, $\|T f\| \geq \limsup \int_{\left|f_{n}\right|>\delta}\left|f_{n}\right| d \lambda$. Since $\limsup \int\left|f_{n}\right| d \lambda=1$ and $\left|f_{n}\right| \leq$ 1 , there exists a subsequence $\left(f_{n_{i}}\right)$ which converges $\lambda$-uniformly to the function 1. Hence $\lim \sup \int_{\left|f_{n}\right|>\delta}\left|f_{n}\right| d \lambda=1$. Consequently, $1 \leq\|T f\|$, and thus $\|T f\|=$ $\|T f\|=1$.

In order to embed other Banach lattices into $\mathbb{W}$ a somewhat more elaborate construction is needed. We will construct lattice homomorphisms from nonclosed sublattices of $\left(\sum \oplus L^{1}\right)_{\ell^{\infty}}$ into Weak $L^{1}$ which are isometries for $\|\cdot\|$ but which are not continuous for $|\|\cdot \mid\|$ (see Remark 1 below), in contrast to the situation of Propositions 1 and 2 .

At first we need a lemma which actually characterizes Weak $L^{1}$ functions on any measure space.

Lemma 1. Let $(X, \Sigma, \mu)$ be a measure space and let $f$ be measurable, $f$ finite a.e. If there exist constants $\gamma$ and $K, K>1$, such that $\int_{K^{i}<|f| \leq K^{i+1}}|f| d \mu \leq \gamma$ for all $i \in \mathbb{Z}$, then $f \in L(1, \infty)$.

Proof. Let $A_{i}=\left\{t: K^{i}<|f(t)| \leq K^{i+1}\right\}$. The condition implies that $K^{i} \mu\left(A_{i}\right) \leq$ $\gamma$. Let $s>0$ be given. Then for some $j \in \mathbb{Z}, K^{j} \leq s<K^{j+1}$. Therefore, $s \mu\{|f|>s\} \leq K^{j+1} \mu\left\{|f|>K^{j}\right\} \leq K^{j+1} \sum_{i=j}^{\infty} \mu\left(A_{i}\right) \leq \gamma K^{2} /(K-1)$. Hence, $f \in L(1, \infty)$.

Theorem 1. Let $E=\left(\sum \oplus L^{1}\right)_{\ell \infty}$ and let $M_{i} \geq 1$ for $i \in \mathbb{N}$. Then the closure of the sublattice $G=\left\{\left(h_{i}\right) \in E: \sup M_{i}^{-1}\left\|h_{i}\right\|_{\infty}<\infty\right\}$ is lattice isometric to a closed sublattice of the Banach envelope of Weak $L^{1}$.

Proof. a) Choose $\varphi: \mathbb{N} \rightarrow \mathbb{N}$ such that for every $i \in \mathbb{N}$ the set $\varphi^{-1}(\{i\})$ is infinite. Let $d_{n}=\max _{1 \leq j \leq n} M_{\varphi(j)}$. Then the operator $S:\left(h_{i}\right) \mapsto\left(f_{n}\right)$, where $f_{n}=h_{\varphi(n)}$, is a lattice isometry from $E$ into itself which maps $G$ into

$$
F=\left\{\left(f_{n}\right): \sup d_{n}^{-1}\left\|f_{n}\right\|_{\infty}<\infty\right\}
$$

Let $X=(0,1] \times(0,1]$ with Lebesgue measure $\mu$. We will construct a lattice homomorphism $T$ from $F$ into the Banach envelope of Weak $L^{1}$ on $X$ with $\|T\| \leq 1$ and $\|T f\| \geq\|f\|$ whenever $f \in S G$.

b) Choose $a_{n}>0$ and $b_{n}>0$ such that

i) $b_{1}=1$,

ii) $a_{n+1}<b_{n+1}<a_{n}<b_{n}$,

iii) $\lim \frac{b_{n}}{a_{n}}=\infty$,

iv) $\ln \frac{a_{l}}{b_{k} d_{k}} \geq \sum_{i=l}^{k} \ln \frac{b_{i}}{a_{i}}$ for $l<k$.

We denote the set $\left\{(s, t): a_{n} \leq s \leq b_{n}, 0<t \leq 1\right\}$ by $A_{n}$.

c) For $f=\left(f_{n}\right) \in F$ and $0<s, t \leq 1$ define

$$
(T f)(s, t)= \begin{cases}\frac{f_{n}(t)}{s} & \text { for }(s, t) \in A_{n}, \\ 0 & \text { for }(s, t) \notin \cup A_{n} .\end{cases}
$$


Obviously, $T$ defines a lattice homomorphism from $F$ into the space of measurable functions on the square $X$. We note that $\int_{A_{n}}|T f| d \mu=\left\|f_{n}\right\| \ln \frac{b_{n}}{a_{n}}$.

d) Let $f=\left(f_{n}\right) \in F$ be given and let $\gamma=\sup d_{n}^{-1}\left\|f_{n}\right\|_{\infty}$. Suppose that there exists $\delta>0$ such that $f_{n}(t) \geq \delta$ for all $n$ and all $t$. Fix $p$ and $q, 0<p<q$, and let $A=\{(s, t): p<T f(s, t) \leq q\}$. We claim that

$$
\int_{A} T f d \mu \leq\|f\| \max \left\{\ln \frac{q}{p}, \ln \frac{q \gamma}{p \delta}\right\} .
$$

We may assume that $A$ is not empty. Let $B_{n}=A \cap A_{n}$. Then $A=\cup B_{n}$. If $(s, t) \in B_{n}$, then $\delta / q \leq f_{n}(t) / q \leq s<f_{n}(t) / p \leq \gamma d_{n} / p$. Let $l$ and $k$ be the minimum and maximum respectively of $\left\{n: B_{n} \neq \Phi\right\}$. We consider two cases:

1) If $l=k$, let $a(t)=\min \left\{\max \left\{a_{k}, \frac{f_{k}(t)}{q}\right\}, b\right\}, \quad b(t)=\max \left\{\min \left\{b_{k}, \frac{f_{k}(t)}{p}\right\}, a\right\}$; then

$$
\begin{aligned}
\int_{A} T f d \mu & =\int_{0}^{1} \int_{a(t)}^{b(t)} \frac{f_{k}(t)}{s} d s d t \\
& \leq \int_{0}^{1} \int_{\frac{f_{k}(t)}{q}}^{\frac{f_{k}(t)}{p}} \frac{f_{k}(t)}{s} d s d t \\
& =\left\|f_{k}\right\| \ln \frac{q}{p} .
\end{aligned}
$$

2) If $l<k$, then $\delta / q \leq b_{k} \leq a_{l} \leq \gamma d_{l} / p \leq \gamma d_{k} / p$. Hence $a_{l} / b_{k} d_{k} \leq q \gamma / p \delta$. Therefore,

$$
\begin{aligned}
\int_{A} T f d \mu & \leq \sum_{n=l}^{k} \int_{B_{n}} T f d \mu \leq \sum_{n=l}^{k}\left\|f_{n}\right\| \ln \frac{b_{n}}{a_{n}} \\
& \leq\|f\| \sum_{n=l}^{k} \ln \frac{b_{n}}{a_{n}} \leq\|f\| \ln \frac{a_{l}}{b_{k} d_{k}} \leq\|f\| \ln \frac{q \gamma}{p \delta}
\end{aligned}
$$

This proves $(\dagger)$. It follows easily from Lemma 1 that $T f \in L(1, \infty)$. Moreover,

$$
\begin{aligned}
\|T f\| & =\limsup _{q / p \rightarrow \infty} \int_{A}|T f| d \mu / \ln \frac{q}{p} \\
& =\limsup _{q / p \rightarrow \infty} \int_{A} T f d \mu / \ln \frac{q}{p} \\
& \leq\|f\| .
\end{aligned}
$$

e) Let $g=\left(g_{n}\right) \in F$ be arbitrary. Choose $\eta>0$ and let $f_{n}=\left(\left|g_{n}\right|+\eta\right)$. Then $f=\left(f_{n}\right) \in F$ and satisfies the assumptions of $\left.\mathrm{d}\right)$. Hence $T f \in L(1, \infty)$ and $\|T f\| \leq\|f\|=\|g\|+\eta$. Since $|T g| \leq T f, T g \in L(1, \infty)$ and $\|T g\| \leq\|g\|+\eta$. Since $\eta>0$ is arbitrary, $\|T g\| \leq\|g\|$. This shows that $T$ maps $F$ into Weak $L^{1}$ and that $\|T f\| \leq\|f\|$ for every $f \in F$.

f) Let $f=\left(f_{n}\right) \in F$ be given. Fix $\alpha$ and $\beta, 0<\alpha<\beta$, and let $p_{n}=\alpha / b_{n}$ and $q_{n}=\beta / a_{n}$. Then

$$
\begin{aligned}
\int_{p_{n}<|T f| \leq q_{n}}|T f| d \mu & \geq \int_{\alpha<\left|f_{n}(t)\right| \leq \beta} \int_{a_{n}}^{b_{n}} \frac{\left|f_{n}(t)\right|}{s} d s d t \\
& =\left(\int_{\alpha<\left|f_{n}(t)\right| \leq \beta}\left|f_{n}(t)\right| d t\right) \ln \frac{b_{n}}{a_{n}} .
\end{aligned}
$$


Since $q_{n} / p_{n}$ tends to $\infty$,

$$
\|T f\| \geq \limsup \int_{\alpha<\left|f_{n}(t)\right| \leq \beta}\left|f_{n}(t)\right| d t .
$$

If $f=S h$, where $h=\left(h_{i}\right) \in G$, then for every $i$ there exist infinitely many $n$ 's with $f_{\varphi(n)}=h_{i}$. Therefore,

$$
\|T S h\| \geq \int_{\alpha<\left|h_{i}(t)\right| \leq \beta}\left|h_{i}(t)\right| d t .
$$

Consequently, $\|T S h\| \geq \sup \left\|h_{i}\right\|=\|h\|$.

g) Finally, let $\psi:(0,1] \rightarrow X$ be a measure-preserving transformation. Then $U: f \mapsto(T S F) \circ \psi$ is a lattice isometry from $G$ into the Banach envelope of Weak $L^{1}$ on $(0,1]$ which can be extended to a lattice isometry from the closure of $G$ into $\mathbb{W}$.

Corollary 1. The sublattice $H=\left\{\left(f_{n}\right):\left\{f_{n}\right\}\right.$ is relatively weakly compact in $\left.L^{1}\right\}$ of $\left(\sum \oplus L^{1}\right)_{\ell \infty}$ is lattice isometric to a closed sublattice of the Banach envelope of Weak $L^{1}$.

Proof. It is easily checked that $H$ is the closure of the sublattice $F=\left\{\left(f_{i}\right)\right.$ : $\left.\sup \left\|f_{i}\right\|_{\infty}<\infty\right\}$ of $\left(\sum \oplus L^{1}\right)_{\ell}^{\infty}$. Now the Corollary follows from Theorem 1 with $M_{i}=1$.

Corollary 2. For every sequence $\left(k_{i}\right)$ of natural numbers, the Banach lattice $\left(\sum \oplus \ell^{1}\left(k_{i}\right)\right)_{\ell^{\infty}}$ is lattice isometric to a closed sublattice of the Banach envelope of Weak $L^{1}$.

Proof. Clearly, for every $i$ there exists a lattice isometry $T_{i}$ from $\ell^{1}\left(k_{i}\right)$ into $L^{1}$ with $\left\|T_{i} x\right\|_{\infty} \leq k_{i}\|x\|$ for all $x \in \ell^{1}\left(k_{i}\right)$. Hence, $T:\left(x_{i}\right) \mapsto\left(T_{i} x_{i}\right)$ is a lattice isometry from $\left(\sum \oplus L^{1}\right)_{\ell^{\infty}}$ into the sublattice $\left\{\left(f_{i}\right): \sup k_{i}^{-1}\left\|f_{i}\right\|_{\infty}<\infty\right\}$ of $\left(\sum \oplus L^{1}\right)_{\ell^{\infty}}$.

Remark 1. The map $T$ from $F$ into Weak $L^{1}$ in the proof of Theorem 2 is not $\||\cdot \||$ continuous (this follows from Theorem 5 below). However, the restriction to any sublattice $F_{1}$ of $F$ will be continuous if $F_{1}$ is closed in $\left(\sum \oplus L^{1}\right)_{\ell \infty}$ (indeed, every positive linear map from a Banach lattice into (Weak $L^{1},\|\| \cdot \|$ ) is continuous). Consequently, if $G_{1}$ is a sublattice of the space $G$ in Theorem 2 and $G_{1}$ is closed in $\left(\sum \oplus L^{1}\right)_{\ell \infty}$, then the restriction of $T S$ is a topological isomorphism from $G_{1}$ into (Weak $L^{1},\left\|\left|\|\mid\|\right.\right.$ ). In particular, the space $\left(\sum \oplus \ell^{1}\left(k_{i}\right)_{\ell \infty}\right)$ is lattice isomorphic to a closed sublattice of (Weak $\left.L^{1},\||\cdot|\|\right)$.

2

A positive element $u$ of a Banach lattice $E$ is called a quasi-interior point if the interval $[0, u]=\{x: 0 \leq x \leq u\}$ is total in $E$. Every separable Banach lattice has a quasi-interior point.

Every Banach lattice is lattice isometric to a closed sublattice of an $\ell^{\infty}$ - product of AL-spaces ([5], Lemma 3.4). We will show that if $E$ is a separable Banach lattice or if $E$ is the dual of a separable Banach lattice and has a quasi-interior point, then $E$ is lattice isometric to a closed sublattice of the Banach lattice $H$ of Corollary 1. 
Lemma 2. Let $E$ be a separable Banach lattice, let $0 \leq u \in E$ be a quasi-interior point, and let $0<x^{\prime} \in E^{\prime}$. Then there exists a lattice homomorphism $T$ from $E$ into $L^{1}$ such that $\|T x\|=\left\langle|x|, x^{\prime}\right\rangle$ for every $x \in E$ and $T u=\left\langle u, x^{\prime}\right\rangle 1$.

Proof. Let $I$ be the closed ideal $\left\{x:\left\langle|x|, x^{\prime}\right\rangle=0\right\}$ and let $F$ be the completion of $E / I$ with the norm $\|x+I\|=\left\langle|x|, x^{\prime}\right\rangle$. Then $F$ is an AL-space and the canonical map $\varphi$ from $E$ into $F$ is a lattice homomorphism with $\|\varphi(x)\|=\left\langle|x|, x^{\prime}\right\rangle$ for every $x \in E$. Thus, $\varphi$ is continuous and therefore $F$ is separable. Moreover, $v=\left\langle u, x^{\prime}\right\rangle^{-1} \varphi(u)$ is a weak order unit of $F$ with $\|v\|=1$. Hence by Kakutani's theorem there is a measure space $(S, \Sigma, \nu), \nu(s)=1$, and a lattice isometry $T_{1}$ from $F$ onto $G=L^{1}(S, \Sigma, \nu)$ which maps $v$ onto the function 1 . Since $G$ is separable, there is a lattice isometry $T_{2}$ from $G$ into $L^{1}$ with $T_{2} 1=1$. Thus, $T=T_{2} \circ T_{1} \circ \varphi$ is the desired lattice homomorphism from $E$ into $L^{1}$.

Theorem 2. Every separable Banach lattice $E$ is lattice isometric to a closed sublattice of the Banach envelope of Weak $L^{1}$.

Proof. We may assume that $E \neq\{0\}$. Since $E$ is separable, $E$ has a quasi-interior point $u$. Also, there is a normalized sequence $\left(x_{i}^{\prime}\right) \subset E^{\prime}, x_{i}^{\prime} \geq 0$, such that $\|x\|=$ $\sup \left\langle|x|, x_{i}^{\prime}\right\rangle$ for every $x \in E$. By Lemma 2, there exist lattice homomorphisms $T_{i}$ : $E \rightarrow L^{1}$ such that $\left\|T_{i} x\right\|=\left\langle|x|, x_{i}^{\prime}\right\rangle$ for every $x \in E$ and $T_{i} u=\left\langle u, x_{i}^{\prime}\right\rangle 1$. Obviously, $x \mapsto\left(T_{i} x\right)$ is then a lattice isometry from $E$ into $\left(\sum \oplus L^{1}\right)_{\ell}$. If $0 \leq x \leq u$, then $0 \leq T_{i} x \leq\left\langle u, x_{i}^{\prime}\right\rangle 1 \leq\|u\| 1$, since $\sup \left\|x_{i}^{\prime}\right\| \leq 1$, and hence $\left\{T_{i} x\right\}$ is relatively weakly compact. Since the interval $[0, u]$ is total in $E, T$ maps $E$ into the sublattice $H$ of $\left(\sum \oplus L^{1}\right)_{\ell}$ of all relatively weakly compact sequences. Now it follows from Corollary 1 that $E$ is lattice isometric to a closed sublattice of the Banach envelope of Weak $L^{1}$.

Let $E$ be a Banach lattice and let $0 \leq x \in E$. The linear hull of the order interval $[0, x]$ is called the principal ideal generated by $x$ and denoted by $E_{x}$.

Lemma 3. Let $E$ be a separable Banach lattice and let $0<x \in E$ and $0 \leq v^{\prime} \in E^{\prime}$. Then there exists a lattice homomorphism $T$ from the closure $G$ of the principal ideal $E_{v^{\prime}}^{\prime}$ into $L^{1}$ with $T v^{\prime}=\left\langle x, v^{\prime}\right\rangle 1$ and $\left\|T y^{\prime}\right\|=\left\langle x,\left|y^{\prime}\right|\right\rangle$ for every $y^{\prime} \in G$.

Proof. By Kakutani's representation theorem for AM-spaces with unit, there exist a compact space $K$ and a lattice isomorphism $S$ from $C(K)$ onto the principal ideal $E_{x}$ with $S 1=x$. Since $S$ is a lattice homomorphism and preserves order intervals, $S^{\prime}$ is a lattice homomorphism from $E^{\prime}$ into the AL-space $C(K)^{\prime}$ with $S^{\prime}\left[0, v^{\prime}\right]=[0, \nu]$ where $\nu=S^{\prime} v^{\prime}$ ([5], Proposition 1.2). Clearly, $\left\|S^{\prime} x^{\prime}\right\|=\left\langle x,\left|x^{\prime}\right|\right\rangle$ for every $x^{\prime} \in E^{\prime}$. Also, $S^{\prime}$ maps the ideal $G$ into the band $L^{1}(K, \nu)$. Since $E$ is separable, $\left[0, v^{\prime}\right]$ is separable for the weak ${ }^{*}$ topology. Hence $[0, \nu]$ is also weak*separable. Now, $[0, \nu]$ is weakly compact, and so weakly separable. Therefore, $L^{1}(K, \nu)$ is separable. Thus there exists a lattice isometry $T_{2}$ from $L^{1}(K, \nu)$ into $L^{1}$ with $T_{2} \nu=\langle x, \nu\rangle 1$. Let $T_{1}$ be the restriction of $S^{\prime}$ to $G$. Then $T=T_{2} \circ T_{1}$ is the desired lattice homomorphism.

Theorem 3. If $E$ is a separable Banach lattice, then the closure of any principal ideal in $E^{\prime}$ is lattice isometric to a closed sublattice of the Banach envelope of Weak $L^{1}$.

Proof. Let $0 \leq v^{\prime} \in E^{\prime}$ be given and let $G$ be the closure of the principal ideal $E_{v^{\prime}}^{\prime}$. Choose $0 \leq x_{i} \in E$ such that $\left\|x^{\prime}\right\|=\sup \left\langle x_{i},\left|x^{\prime}\right|\right\rangle$ for every $x^{\prime} \in E^{\prime}$. By Lemma 
3, there exist lattice homomorphisms $T_{i}$ from $G$ into $L^{1}$ with $T_{i} v^{\prime}=\left\langle x_{i}, v^{\prime}\right\rangle 1$ and $\left\|T_{i} x^{\prime}\right\|=\left\langle x_{i},\left|x^{\prime}\right|\right\rangle$ for every $x^{\prime} \in G$. As in the proof of Theorem 2, it follows easily that $T: x^{\prime} \mapsto\left(T_{i} x^{\prime}\right)$ is a lattice isometry from $G$ into the sublattice $W$ of $\left(\sum \oplus L^{1}\right)_{\ell_{\infty}}$ of all relatively weakly compact sequences. Now, Corollary 1 implies that $G$ is lattice isometric to a closed sublattice of the Banach envelope of Weak $L^{1}$.

Corollary 3. Let $E$ be a separable Banach lattice. If the dual $E^{\prime}$ has a quasiinterior point, then $E^{\prime}$ is lattice isometric to a closed sublattice of the Banach envelope of Weak $L^{1}$.

Corollary 4. Let $(S, \Sigma, \nu)$ be a separable measure space. Then the Banach lattice $L^{\infty}(S, \Sigma, \nu)$ is lattice isometric to a closed sublattice of the Banach envelope of Weak $L^{1}$.

Of course, Corollary 4 also follows from Proposition 2.

Let $(S, \Sigma, \nu)$ be a measure space. The space Weak $L^{p}$ consists of all (equivalence classes of) measurable functions $f$ on $S$ for which

$$
\sup _{s>0} s(\nu\{|f|>s\})^{1 / p}<\infty .
$$

If $1<p<\infty$, then Weak $L^{p}$ with the norm

$$
\|f\|=\sup _{0<\nu(B)<\infty} \nu(B)^{1 / p-1} \int_{B}|f| d \nu
$$

is a dual Banach lattice, which we denote by $L(p, \infty, \nu)$. Except in the finite dimensional case, this space does not have a quasi-interior point.

Theorem 4. Let $(S, \Sigma, \nu)$ be a separable measure space and let $1<p<\infty$. Then Weak $L^{p}$ is lattice isometric to a closed sublattice of the Banach envelope of Weak $L^{1}$.

Proof. At first we consider the real interval $(0, \alpha), 0<\alpha \leq \infty$, with Lebesgue measure $\mu$. Since the predual $L(p /(p-1), 1)$ is separable, there is a sequence $\left(B_{n}\right)$ of measurable subsets of $(0, \alpha)$ such that

$$
\|f\|=\sup _{n} \mu\left(B_{n}\right)^{1 / p-1} \int_{B_{n}}|f| d \mu, \quad f \in L(p, \infty, \mu) .
$$

For each $n$ there exists a bijection $\psi_{n}$ from $(0,1]$ onto $B_{n}$ such that $A \subset(0,1]$ is measurable if and only if $\psi_{n}(A)$ is measurable and such that $\mu\left(\psi_{n}(A)\right)=\lambda(A) \mu\left(B_{n}\right)$. An easy calculation shows that for every measurable subset $A \subset(0,1]$,

$$
\begin{aligned}
\mu\left(B_{n}\right)^{1 / p} \int_{A}\left|f \circ \psi_{n}\right| d \lambda & =\mu\left(B_{n}\right)^{1 / p-1} \int_{\psi_{n}(A)}|f| d \mu \\
& \leq \mu\left(B_{n}\right)^{1 / p-1} \mu\left(\psi_{n}(A)\right)^{1-1 / p}|| f|| \\
& \leq \lambda(A)^{1-1 / p}\|f\| .
\end{aligned}
$$

This shows that $\left\{\mu\left(B_{n}\right)^{1 / p} f \circ \psi_{n}\right\}$ is relatively weakly compact in $L^{1}$. Also, the first equation with $A=(0,1]$ shows that $f \mapsto\left(\mu\left(B_{n}\right)^{1 / p} f \circ \psi_{n}\right)$ is a lattice isometry from Weak $L^{p}$ into $\left(\sum \oplus L^{1}\right)_{\ell^{\infty}}$. It follows from Corollary 1 that Weak $L^{p}$ is lattice isometric to a closed sublattice of the Banach envelope of Weak $L^{1}$. Now let $(S, \Sigma, \nu)$ be separable and $\sigma$-finite and let $\alpha=\nu(S)$. Then $(S, \Sigma, \nu)$ is isomorphic to a $\sigma$-subalgebra $\Sigma_{1}$ of the real interval $(0, \alpha)$ with Lebesgue measure $\mu$. It is 
clear that $L(p, \infty, \nu)$ is lattice isomorphic to the sublattice of all $\Sigma_{1}$-measurable functions of $L(p, \infty, \mu)$. It follows from Lemma 8 of [6] that this isomorphism is an isometry.

Remark 2. Let $1<p<\infty$ and let $1 / p+1 / q=1$. The inclusion map from $L^{p}(0,1]$ into Weak $L^{p}$ on $(0,1]$ is a lattice isomorphism but not an isometry. For $f \in L^{p}$, define $T f$ by

$$
(T f)(s, t)=q f(t) / s^{1 / p}, \quad(s, t) \in X=(0,1] \times(0,1] .
$$

Let $\varphi:(0,1] \rightarrow X$ be a measure-preserving transformation. Then $f \mapsto(T f) \circ \varphi$ is a lattice isometry from $L^{p}$ into the Lorentz space $L(p, \infty, \lambda)$.

3

It follows from Theorem 2 that there is a lattice isometry from $\left(\sum \oplus L^{1}\right)_{c_{0}}$ into the Banach envelope of Weak $L^{1}$. However, no such embedding factors through Weak $L^{1}$ as we will see. This shows that the operator $T$ in the proof of Theorem 2 is not a continuous operator into the quasinormed space Weak $L^{1}$.

Lemma 4. Let $0<f \in$ Weak $L^{1}$ with $\|f\|>1$. Then for every $\alpha>1$ there exists $p>0$ such that

$$
\int_{p<f \leq \alpha p} f d \lambda \geq \ln \alpha
$$

Proof. Choose $k \in \mathbb{N}$ such that $\|f\|>(k+1) / k$. Then there exist $0<r<q$ such that $\alpha^{k} \leq q / r$ and $(k+1) / k \ln (q / r) \leq \int_{r<f \leq q} f d \lambda$. Let $m=[\ln (q / r) / \ln \alpha]$. Then $m \geq k$ and

$$
\begin{aligned}
(m+1) \ln \alpha & \leq\left(1+\frac{1}{k}\right) m \ln \alpha \leq\left(1+\frac{1}{k}\right) \ln \frac{q}{r} \\
& \leq \int_{r<f \leq q} f d \lambda \leq \int_{r<f \leq \alpha^{m+1} r} f d \lambda=\sum_{i=0}^{m} \int_{\alpha^{i} r<f \leq \alpha^{i+1} r} f d \lambda \\
& \leq(m+1) \max \left\{\int_{\alpha^{i} r<f \leq \alpha^{i+1} r} f d \lambda\right\} .
\end{aligned}
$$

Hence, for some $j, \ln \alpha \leq \int_{\alpha^{j} r<f \leq \alpha^{j+1} r} f d \lambda$. Now let $p=\alpha^{j} r$.

Lemma 5. Let $T$ be a lattice homomorphism from $L^{1}$ into Weak $L^{1}$ and let $0 \leq$ $f \in L^{1}$ and $p<q$ be given. Suppose that $\int_{p<T f \leq q} T f d \lambda>0$. Then for every $\gamma \geq 1$ there exists $0 \leq g \in L^{1}$ with $\|g\| \leq\|f\|$ and

$$
\int_{\gamma p<T g \leq \gamma q} T g d \lambda=\int_{p<T f \leq q} T f d \lambda .
$$

Proof. The assertion is trivial if $\gamma=1$. So we assume that $\gamma>1$. Let $D=\{p<$ $T f \leq q\}$. For every $\lambda$-measurable set $A$ let $\nu(A)=\int_{D} T\left(f \chi_{A}\right) d \lambda$. Since $T$ is a continuous map from $L^{1}$ into Weak $L^{1}$ (indeed, $\left\|f_{i}\right\| \leq 2^{-2 i}$ implies $\left|T f_{n}\right| \leq$ $\left.2^{-n} T\left(\sum 2^{i}\left|f_{i}\right|\right)\right), T\left(f \chi_{A}\right)$ tends to zero in measure as $\lambda(A)$ tends to zero. By the dominated convergence theorem $\int_{D} T\left(f_{A}\right) d \lambda$ tends to zero as $\lambda(A)$ tends to zero. Hence $\nu$ is a measure which is absolutely continuous with respect to $\lambda$.

Therefore, there exists $0 \leq h_{1} \in L^{1}$ with $\nu(A)=\int_{A} h_{1} d \lambda$. It is clear that $h_{1}$ vanishes $\lambda$-almost everywhere on the set $\{f=0\}$. Let $h(t)=h_{1}(t) / f(t)$ if $f(t)>0$ 
and $h(t)=0$ elsewhere. Then $\nu(A)=\int_{A} h f d \lambda$ for every measurable set $A$. Let $\beta=\inf \left\{\alpha: \int_{h>\alpha} h f d \lambda \leq \gamma^{-1}\|\nu\|\right\}$. Then $\int_{h>\beta} h f d \lambda \leq \gamma^{-1}\|\nu\| \leq \iint_{h \geq \beta} h f d \lambda$. There exists a measurable set $B$ with $\{h>\beta\} \subset B \subset\{h \geq \beta\}$ and $\int_{B} h f d \lambda=$ $\gamma^{-1}\|\nu\|$. Then $\beta \int_{B} f d \lambda \leq \gamma^{-1}\|\nu\|$ and $\left(1-\gamma^{-1}\right)\|\nu\| \leq \beta\left(\|f\|-\int_{B} f d \lambda\right)$, and so $\int_{B} f d \lambda /\left(\|f\|-\int_{B} f d \lambda\right) \leq \gamma^{-1} /\left(1-\gamma^{-1}\right)$. This easily implies that $\int_{B} f d \lambda \leq$ $\gamma^{-1}\|f\|$. Let $g=\gamma f \chi_{B}$. Then $\|g\| \leq\|f\|$ and

$$
\int_{p<T f \leq q} T g d \lambda=\gamma \nu(B)=\gamma \int_{B} h f d \lambda=\|\nu\|=\int_{p<T f \leq q} T f d \lambda .
$$

Since $T$ is a lattice homomorphism, $T\left(f \chi_{B}\right)$ and $T\left(f \chi_{\mathfrak{C}_{B}}\right)$ are disjoint. Hence

Therefore

$$
\int_{p<T\left(f \chi_{B}\right) \leq q} T\left(f \chi_{B}\right) d \lambda=\int_{p<T f \leq q} T\left(f \chi_{B}\right) d \lambda
$$

$$
\int_{\gamma p<T g \leq \gamma q} T g d \lambda=\gamma \int_{p<T f \leq q} T\left(f \chi_{B}\right) d \lambda=\int_{p<T f \leq q} T f d \lambda .
$$

Theorem 5. Let $T$ be a lattice homomorphism from $\left(\sum \oplus L^{1}\right)_{c_{0}}$ into Weak $L^{1}$. Then $T$ as a map into the Banach envelope of Weak $L^{1}$ factors through $L^{1}$.

Proof. For $i \in \mathbb{N}$ define $\varphi_{i}: L^{1} \rightarrow\left(\sum \oplus L^{1}\right)_{c_{0}}$ by $\varphi_{i}(f)=\left(f_{n}\right)$, where $f_{n}=f$ if $n=i$ and $f_{n}=0$ otherwise, and let $T_{i}=t \circ \varphi_{i}$. We may assume that $\left\|T_{i}\right\|>0$ for all $i$. Suppose that $\sum\left\|T_{i}\right\|=\infty$. Then there exist $0 \leq f_{i} \in L^{1}, \lim \left\|f_{i}\right\|=0$, with $\sum\left\|T_{i} f_{i}\right\|=\infty$. Let $\left\{A_{n}\right\}$ be a partition of $\mathbb{N}$ into finite subsets such that $\sum_{i \in A_{n}}\left\|T_{i} f_{i}\right\|>n$ for every $n$.

By Lemma 4 , for every $i \in A_{n}$ we can choose $p_{i}>0$ such that $\int_{p_{i}<T_{i} f_{i} \leq e^{n} p_{i}} T_{i} f_{i}>$ $n\left\|T_{i} f_{i}\right\|\left(1-n^{-1}\right)$. Let $r_{n}=\max \left\{p_{i}: i \in A_{n}\right\}$. By Lemma 5 , for $i \in A_{n}$ there exists $0 \leq g_{i} \in L^{1},\left\|g_{i}\right\| \leq\left\|f_{i}\right\|$, such that $\int_{r_{n}<T_{i} f_{i} \leq e^{n} r_{n}} T_{i} g_{i}>n\left\|T_{i} f_{i}\right\|\left(1-n^{-1}\right)$. Let $g=\left(g_{i}\right)$. Then $g \in F, T g=\sum T_{i} g_{i}$, and the functions $T_{i} g_{i}$ are pairwise disjoint. Therefore,

$$
\begin{aligned}
\int_{r_{n}<T g \leq e^{n} r_{n}} T g d \mu & =\sum \int_{r_{n}<T_{i} g_{i} \leq e^{n} r_{n}} T_{i} g_{i} d \mu \\
& \geq \sum_{i \in A_{n}} \int_{r_{n}<T_{i} g_{i} \leq e^{n} r_{n}} T_{i} g_{i} d \mu \geq n(n-1) .
\end{aligned}
$$

Obviously, this contradicts the fact that

$$
\limsup _{q / p \rightarrow \infty} \int_{p<T g \leq q} T g d \mu / \ln \frac{q}{p}<\infty .
$$

Thus, $\sum\left\|T_{i}\right\|<\infty$. Let $G$ be the completion of $F$ for the norm $\left\|\left(f_{i}\right)\right\|_{1}=$ $\sum\left\|T_{i}\right\|\left\|f_{i}\right\|$. Then $G$ is isometric to $L^{1}$ and $T$ as a map into $\mathbb{W}$ factors through G.

A positive linear operator $T$ from a Banach lattice $E$ into a Banach lattice $F$ is called an order isometry if $T x \geq 0$ implies $x \geq 0$ and if $\|T x\|=\|x\|$ for all $x \in E$. It follows from Theorem 5 that no lattice isometry from $\left(\sum \oplus L^{1}\right)_{\ell^{\infty}}$ into the Banach envelope of Weak $L^{1}$ factors through Weak $L^{1}$ by a lattice homomorphism. 
However, there is an order isometry from $\left(\sum \oplus L^{1}\right)_{\ell^{\infty}}$ into the Banach envelope of Weak $L^{1}$.

Theorem 6. The Banach lattice $\left(\sum \oplus L^{1}\right)_{\ell \infty}$ is order isometric to a closed subspace of the Banach envelope of Weak $L^{1}$.

Proof. For $f \in L^{1}$ let $P_{n} f$ be the conditional expectation of $f$ with respect to the $\sigma$-subalgebra generated by the intervals $\left((j-1) 2^{-n}, j 2^{-n}\right]$. We may also consider $P_{n}$ as a positive operator from $L^{1}$ into $\ell^{1}\left(2^{n}\right)$. Since the $P_{n}$ 's are positive contractions and $P_{n} f$ converges to $f$ in $L^{1}$, the map $f \mapsto\left(P_{n} f\right)$ is an order isometry from $L^{1}$ into $\left(\sum \oplus L^{1}\right)_{\ell \infty}$. Then also $T:\left(f_{i}\right) \mapsto\left(P_{n} f_{i}\right)$ is an order isometry from $E=$ $\left(\sum \oplus L^{1}\right)_{\ell \infty}$ into $F=\left(\sum \oplus \ell^{1}\left(k_{(i, n)}\right)\right)_{\ell \infty}$, where $k_{(i, n)}=2^{n}$ for every $(i, n) \in \mathbb{N} \times \mathbb{N}$. By Corollary 2, there is a lattice isometry $S$ from $F$ into the Banach envelope of Weak $L^{1}$. Hence $S \circ T$ is an order isometry from $E$ into the Banach envelope of Weak $L^{1}$.

Corollary 5. Let $E$ be a separable Banach lattice and let $0<x^{\prime} \in E^{\prime}$. Then the band generated by $x^{\prime}$ in $E^{\prime}$ is lattice isometric to a closed sublattice of $\left(\sum \oplus L^{1}\right)_{\ell \infty}$.

Proof. A slight modification of the proofs of Lemma 3 and Theorem 3 shows that the band generated by $x^{\prime}$ is lattice isometric to a closed sublattice of $\left(\sum \oplus L^{1}\right)_{\ell \infty}$.

Corollary 6. Let $E$ be a separable Banach lattice. If $E^{\prime}$ has a weak order unit, then $E^{\prime}$ is order isometric to a closed subspace of the Banach envelope of Weak $L^{1}$.

Corollary 7. Let $E$ be a separable Banach lattice. If $E$ does not contain a closed subspace order isometric to $C(\Delta), \Delta$ the Cantor set, then the dual $E^{\prime}$ is order isometric to a closed subspace of the Banach envelope of Weak $L^{1}$.

Proof. Since $E$ is separable, it follows easily from Theorem 2 of [8] that $E^{\prime}$ has a weak order unit if and only if $E$ does not contain a closed subspace order isometric to $C(\Delta)$.

Remark 3. It follows from Remark 1 that the spaces in Theorem 6 and Corollaries 5,6 , and 7 are order isomorphic (and thus topologically isomorphic) to closed subspaces of (Weak $\left.L^{1},\|\cdot\| \mid \|\right)$.

\section{REFERENCES}

1. M. Cwikel and C. Fefferman, Maximal seminorms on Weak $L^{1}$, Studia Math. 69 (1980), 149-154. MR 83b:46033

2. M. Cwikel and C. Fefferman, The canonical seminorm on Weak $L^{1}$, Studia Math. 78 (1984), 275-278. MR 86i:46030

3. N. J. Kalton, Banach space embeddings into $L_{0}$, Israel J. Math 52 (1985), 305-319. MR 87k:46045

4. J. Kupka and N. T. Peck, The $L_{1}$ structure of Weak $L^{1}$, Math. Ann. 269 (1984), 235-262.

5. H. P. Lotz, Extensions and liftings of positive linear mappings on Banach lattices, Trans. Amer. Math. Soc. 211 (1975), 85-100. MR 52:4022

6. H. P. Lotz, Weak* convergence in the dual of Weak $L^{p}$, Israel J. Math. (to appear).

7. H. P. Lotz, On the dual of the space Weak $L^{1}$.

8. H. P. Lotz and H. P. Rosenthal, Embeddings of $C(\Delta)$ and $L^{1}[0,1]$ in Banach lattices, Israel J. Math. 31 (1978), 169-179. MR 80g:46023

9. N. T. Peck and M. Talagrand, Banach sublattices of weak $L_{1}$, Israel J. Math 59 (1987), 257-271. MR 89a:46045

Department of Mathematics, University of Illinois, Urbana, Illinois 61801 